\title{
The comparative effectiveness of statin therapy in selected chronic diseases compared with the remaining population
}

\author{
Xia Sheng ${ }^{1}$, Michael J Murphy ${ }^{2}$, Thomas M MacDonald ${ }^{1}$ and Li Wei ${ }^{{ }^{*}}$
}

\begin{abstract}
Background: Total cholesterol (TC) concentration is the most commonly used measure of statin efficacy in the UK. This study aimed to evaluate the effectiveness of statins in lowering TC, cardiovascular events (CV) and mortality five common chronic diseases (chronic obstructive pulmonary disease (COPD), osteoarthritis (OA), rheumatoid arthritis (RA), chronic kidney disease (CKD), and diabetes mellitus (DM)) and to compare effectiveness with the rest of the population not recorded as having these diseases.
\end{abstract}

Methods: A population-based cohort study was conducted in Tayside population who had at least two TC measurements between 1993 and 2007. There were 12,140 patients with chronic diseases and 9,481 patients in the rest of the population not recorded as having these chronic diseases. The main outcomes were TC change from baseline, CV events and all-cause mortality.

Results: Statin-associated TC reductions varied from 15\% to $28 \%$ with baseline value of between 5.1 and $5.9 \mathrm{mmol} / \mathrm{L}$ in the primary prevention (PP) and from $7 \%$ to $23 \%$ with baseline value of 4.5 to $5.2 \mathrm{mmol} / \mathrm{L}$ in the secondary prevention (SP) among chronic diseases patients. In the rest of the population, TC reductions with statins were $31 \%$ in PP and $28 \%$ in SP with baselines of $6.3 \mathrm{mmol} / \mathrm{L}$ and $5.3 \mathrm{mmol} / \mathrm{L}$, respectively (test of heterogeneity with chronic disease groups: $\mathrm{p}<0.001)$. A notional reduction of $0.5 \mathrm{mmol} / \mathrm{L}$ in $\mathrm{TC}$ predicted variable reductions in incident $\mathrm{CV}$ events of 30\% in RA, 19\% in CKD, and 20\% in DM, and recurrent CV events by $62 \%$ in COPD, $16 \%$ in CKD, and 19\% in DM. The corresponding figures for the rest of population were $12 \%$ for incident CV events and $17 \%$ for the recurrent CV events, respectively. Risk reductions for all-cause mortality varied from $20 \%$ to $36 \%$ in PP and from 18\% to $40 \%$ in $\mathrm{SP}$, except in OA or RA patients in the chronic diseases and $11 \%$ in PP and $16 \%$ in the rest of population (test of heterogeneity: $p>0.05$ ).

Conclusions: The effectiveness of statins in common chronic diseases varied. With the exception of diabetes, statins tends to be less effective in patients with the chronic diseases compared with the rest of the study population. Changes in TC with statins appear not to correlate well with the changes in cardiovascular events and all-cause mortality.

Keywords: Statins, Total cholesterol, Cardiovascular, Mortality, Chronic diseases

\footnotetext{
* Correspondence: li@memo.dundee.ac.uk

${ }^{1}$ Medicines Monitoring Unit, Division of Medical Sciences, Ninewells Hospital

\& Medical School, University of Dundee, Dundee DD1 9SY, UK

Full list of author information is available at the end of the article
} 


\section{Background}

Chronic disease now accounts for the majority of global morbidity and mortality [1,2]. The prevalence of chronic obstructive pulmonary disease (COPD) is $9-10 \%$ based on spirometry testing [3] and is expected to be the fourth leading cause of death in the world by $2030[4,5]$. At least $2.8 \%$ of the population (171 million) in the world suffer from diabetes mellitus (DM) in 2000 and this number is estimated to double by 2030 [6]. Chronic kidney disease (CKD) affects over 500 million people worldwide [7]. The prevalence of CKD is $7.2 \%$ in those aged 30 years or older and varies from $23.4 \%$ to $35.8 \%$ in persons aged 64 years or older [8]. Rheumatoid arthritis (RA) has an estimated prevalence of $0.5-1.0 \%$ [9]. Osteoarthritis (OA) is the most common form of arthritis and the leading cause of chronic disability worldwide [10]. The prevalence of OA increases with age and $12 \%$ of people over the age of 65 have symptomatic knee OA [11]. In a significant proportion of patients with these chronic diseases, statins would be indicated for the prophylaxis of increased cardiovascular (CV) risk [12-21]. Certainly, dyslipidemia is associated with an increased CV risk in patients with chronic diseases [22-24].

The ability of statins to reduce cardiovascular risk by $21 \%$ per mmol of low density lipoprotein cholersterol (LDL-C) reduction was established in trial populations [25]. However, in the UK, clinicians usually make treatment decisions based on total cholesterol (TC), rather than LDL cholesterol (although the latter can in certain circumstances be calculated), sometimes using high density lipoprotein concentration (HDL-C) measurements as well. We have previously shown that TC reduction with lipid-lowering drugs predicts outcomes in the statin trials almost as well as LDL-C and that TC can be used as a reasonable measure of statin efficacy in the absence of LDL-C [26,27]. We set out to compare the effect of statins on total cholesterol-lowering, CV events, and all-cause mortality in patients with five chronic diseases (COPD, OA, RA, CKD, or DM) using the rest of the population who had no record of having these diseases as the referent group.

\section{Methods}

We performed a population-based longitudinal study using the Medicines Monitoring (MEMO) unit recordlinkage database in Tayside, Scotland. This study was approved by Tayside Research Ethics Committee and the Tayside Caldicott Guardians. The study population consisted of residents of Tayside who were registered with a general practitioner between January 1993 and December 2007 and remained residents in Tayside or died during the study period. Study subjects were the Tayside population who had at least two different TC measurements separated by at least 30 days of follow-up. They were divided into chronic disease groups and the rest of the population who did not have these chronic diseases. The chronic disease groups included patients with a primary diagnosis of COPD, OA, RA, CKD, or DM between 1993 and 2007. COPD patients were identified from The Tayside Allergy and Respiratory Disease Information system (TARDIS) and Scottish Morbidity Record (SMR01) which are centralised records of all Scottish hospital admission diagnoses; OA or RA patients were identified from the regional Arthritis dataset, SMR01, and patients with disease-modifying anti-rheumatic drugs (DMARDs) use in the prescription database; CKD patients were identified from SMR01 and the regional biochemistry database (serum creatinine of $220 \mu \mathrm{mol} / \mathrm{L}$ or higher); DM patients were identified from Diabetes Audit and Research in Tayside Scotland (DARTS) database which is derived from the Scottish care information diabetes collaboration (SCI-DC) [28]. SMR1 data were available from 1989 onward. The date of their first diagnosis of each disease was used as the entry date in chronic disease group. A frequency-matched calendar year method was used to allocate an entry date for the rest of the population. TC measurements were obtained from the regional biochemistry database. Subjects were categorized into statin-exposed and statin-unexposed groups according to statin use status during the follow-up. Subjects were also classified into primary prevention (PP) and secondary prevention (SP) cohorts according to whether they had established $\mathrm{CV}$ disease prior to the entry date.

The main outcomes were TC change calculated as TC at the baseline minus $\mathrm{TC}$ at the end of follow up, the incident (new) or recurrent Anti-platelet Trialist's Collaboration (APTC) events of non-fatal myocardial infarction (MI), non-fatal stroke, or vascular death, the individual components of APTC, and all-cause mortality during the follow-up. All-cause mortality data were obtained from the General Register Office for Scotland. Cox regression models with a time-dependent variable of statins to avoid immoral time bias were employed to assess the risk of APTC events or all-cause mortality and adjusted for potential confounders including age, gender, socioeconomic status, TC concentration change, co-morbidities, and concurrent use of medications. Cox model assumptions were checked prior to analysis. To determine the presence of heterogeneity across different study populations (different chronic diseases and the rest of the population), the heterogeneity test (Cochrane's $X^{2}$ test (Q-statistics) was performed [29]. 'Dose equivalents' of simvastatin was used for other statin treatments in order to calculate the mean daily dose. All analyses were carried out using SAS version 9.1.

\section{Results}

Statins in chronic diseases

In total, 9,955 patients in the PP cohort $(6,037$ in the statin-exposed group and 3,918 in the statin-unexposed 
group) and 2,185 patients in the SP cohort (1,427 in the statin-exposed group and 758 in the statin-unexposed group). The PP cohort consisted of 1,274 COPD, 1,269 OA, 430 RA, 998 CKD, and 5,984 DM patients, in which 617 (48.4\%), 696 (54.8\%), 181 (42.1\%), 442 (44.3\%), and $4,101(68.5 \%)$ were in the statin-exposed groups, respectively. The SP cohort included 443 COPD, 247 OA, 78 RA, 704 CKD, and $713 \mathrm{DM}$ patients, in which there were 292 (65.9\%), 175 (70.9\%), 60 (76.9\%), 386 (54.8\%), and $514(72.1 \%)$ in the statin-exposed groups, respectively. The mean follow-up years in PP were 3.08 in COPD patients, 3.61 in OA patients, 3.90 in RA patients, 3.61 in CKD patients, and 4.65 in DM patients in the statin-exposed groups, and were 2.51, 2.69, 3.14, 2.51 , and 3.50 in the statin-unexposed groups, respectively. Correspondingly, the mean follow-up periods in SP were $3.05,2.88,3.15,2.92$, and 4.32 in the statinexposed group, and were 2.26, 2.72, 2.72, 1.91, and 3.13 in the statin-unexposed groups.

Table 1 shows the baseline characteristics of subjects. TC concentrations ranged from $5.11 \mathrm{mmol} / \mathrm{L}$ to $5.90 \mathrm{mmol} / \mathrm{L}$ in $\mathrm{PP}$ and from $4.54 \mathrm{mmol} / \mathrm{L}$ to $5.20 \mathrm{mmol} / \mathrm{L}$ in $\mathrm{SP}$ $(\mathrm{P}<0.001$ between chronic diseases in both $\mathrm{PP}$ and $\mathrm{SP})$. Statin-associated TC reductions varied from $15 \%$ to $28 \%$ in PP and from $7 \%$ to $23 \%$ in SP (Figure 1). Diabetic patients had the most pronounced TC reduction with statins irrespective of PP or SP. TC changes in the statin-unexposed groups were much lower than those in the statin-exposed groups in both PP and SP (Figure 1).

The crude event rates per 1000 person-years (PYs) for each outcome are shown in Additional file 1: Appendix 1. CKD patients taking statins had the highest event rates for most outcomes in both PP and SP. DM patients taking statins had the lowest event rates in PP and OA patients taking statins had the lowest event rates in SP.

Figure 2 shows the predicted proportional hazard ratio for each outcome per notional $0.5 \mathrm{mmol} / \mathrm{L}$ statinassociated TC reduction (in order to avoid negative numbers, hazards ratios are given per $0.5 \mathrm{mmol} / \mathrm{L}$ TC reduction). For the incident APTC events, $0.5 \mathrm{mmol} / \mathrm{L}$ TC reduction with statins translated into $30 \%$ reduction in RA patients, $20 \%$ in DM patients, and $19 \%$ in CKD patients, but no effect in COPD or OA patients. For recurrent APTC events, a $0.5 \mathrm{mmol} / \mathrm{L}$ TC fall resulted in $62 \%$ reduction in COPD, $19 \%$ in DM, and $16 \%$ in CKD, but no effect in OA or RA (Figure 3). Risk reduction in incident non-fatal MI or incident non-fatal stroke was observed in DM patients (both 18\%), but was not seen in other chronic disease patients. Risk of recurrent nonfatal MI was reduced by $30 \%$ in CKD and $24 \%$ in DM. Risk of recurrent non-fatal stroke was reduced by $35 \%$ in CKD patient. Risk reduction of CV mortality was 37\% in $\mathrm{OA}, 20 \%$ in $\mathrm{CKD}$, and $19 \%$ in $\mathrm{DM}$ in $\mathrm{PP}$, and $65 \%$ in COPD, $24 \%$ in CKD, and $25 \%$ in DM in SP (Additional file 1: Appendix 2). A $0.5 \mathrm{mmol} / \mathrm{L}$ TC reduction was associated with reductions in all-cause mortality of about $23 \%$ in COPD, 36\% in OA, 31\% in RA, $27 \%$ in CKD, and $20 \%$ in DM in PP, and $40 \%$ in COPD, $26 \%$ in CKD, and $18 \%$ in DM in SP (Figure 2).

\section{Statins in the rest of the population}

7,964 subjects $(4,574$ statin-exposed and 3,390 statin-unexposed) were included in PP with a mean follow up of 3.07 years in the statin-exposed group and 2.65 in the statin-unexposed group and 1,517 subjects (1,315 statinexposed and 202 statin-unexposed) in SP with a mean follow up of 2.89 and 2.37 years, respectively. Statinassociated TC concentration fell by $31 \%$ from baseline of $6.03 \mathrm{mmol} / \mathrm{L}$ in $\mathrm{PP}$ and $28 \%$ from $5.28 \mathrm{mmol} / \mathrm{L}$ in SP (Figure 1). Approximately $12 \%$ of TC reduction was also observed in the statin-unexposed group.

The crude event rates per 1000 PYs were 14.8 (95\% CI 12.9-16.9) for incident APTC events and 43.4 (95\%CI 37.3-50.6) for recurrent APTC events in the statinexposed group. And the crude mortality rate were 12.2 (95\%CI 10.5-14.2) in PP and 33.8 (95\%CI 28.4-40.3) in SP (Additional file 1: Appendix 1). Statin treatment reduced APTC events, APTC components, or all-cause mortality by approximately $12 \%$ in $\mathrm{PP}$ and by approximately $17 \%$ in SP per $0.5 \mathrm{mmol} / \mathrm{L} \mathrm{TC}$ reduction (Figure 2 and Additional file 1: Appendix 2).

\section{Comparison between chronic disease patients and the rest of the population}

Baseline TC concentrations in chronic disease patients were lower than those in the rest of the population in the statin-exposed groups (both PP and SP: $\mathrm{P}<0.001$ ). Compared with chronic diseases patients, the $\mathrm{mmol} / \mathrm{L}$ fall of TC concentration and the percentage fall of TC with statin use were larger in the remaining population (both PP and SP: P<0.001). The heterogeneity of the proportional risk reduction in incident APTC events $(\mathrm{p}<0.01)$ and all-cause mortality in PP $(\mathrm{p}<0.0001)$ per $0.5 \mathrm{mmol} / \mathrm{L}$ TC reduction was present across chronic disease patients and the rest of the population, but not in recurrent APTC events $(\mathrm{p}=0.53)$, incident non-fatal MI $(p=0.66)$, recurrent non-fatal MI $(p=0.39)$, incident non-fatal stroke $(\mathrm{p}=0.60)$, recurrent non-fatal stroke $(\mathrm{p}=0.10), \mathrm{CV}$ mortality in PP $(\mathrm{p}=0.16), \mathrm{CV}$ mortality in $\mathrm{SP}(\mathrm{p}=0.22)$, and all-cause mortality in SP $(\mathrm{p}=0.12)$.

\section{Discussion}

We have examined the effectiveness of statins in chronic disease patients compared with the remainder of the population. With the exception of DM, statin-associated $\mathrm{TC}$ reductions in chronic disease patients were in general smaller than those in the rest of the population. Use of statins was associated with improved survival in these 
Table 1 Baseline characteristics in chronic diseases patients and the rest of the population

\begin{tabular}{|c|c|c|c|c|c|c|c|c|c|c|c|c|}
\hline & \multicolumn{2}{|c|}{ COPD n (\%) } & \multicolumn{2}{|c|}{ OA n (\%) } & \multicolumn{2}{|c|}{ RA n (\%) } & \multicolumn{2}{|c|}{ CKD n (\%) } & \multicolumn{2}{|c|}{ DM n (\%) } & \multicolumn{2}{|c|}{$\begin{array}{c}\text { The rest of the population } \\
\mathrm{n}(\%)\end{array}$} \\
\hline & $P P$ & $S P$ & $P P$ & $S P$ & $P P$ & $S P$ & $P P$ & $S P$ & $P P$ & $S P$ & $P P$ & $S P$ \\
\hline Statin-exposed & $617(48.4)$ & 292(65.9) & $696(54.8)$ & 175(70.9) & $181(42.1)$ & $60(76.9)$ & $442(44.3)$ & $386(54.8)$ & $4101(68.5)$ & $514(72.1)$ & $4574(57.4)$ & 1315(86.6) \\
\hline Age (years) (mean SD) & $68.5(8.8)$ & $70.2(9.0)$ & $68.5(8.9)$ & 70.8(9.6) & $63.9(11.5)$ & $68.1(9.9)$ & $66.7(14.1)$ & $73.6(10.5)$ & $60.9(11.8)$ & $67.3(10.5)$ & $64.2(11.1)$ & $67.1(10.9)$ \\
\hline Male & 297(48.1) & 169(57.9) & $295(42.5)$ & $95(54.6)$ & $46(25.4)$ & $29(48.3)$ & $248(56.4)$ & $239(62.2)$ & 2079(51.1) & $323(63.0)$ & $2182(47.7)$ & $824(62.7)$ \\
\hline $\begin{array}{l}\text { Baseline TC }(\mathrm{mmol} / \mathrm{L}) \\
\text { (mean SD) }\end{array}$ & $5.30(1.20)$ & $4.68(1.10)$ & $5.30(1.19)$ & $4.54(1.07)$ & $5.54(1.10)$ & $4.95(1.28)$ & $5.11(1.40)$ & $4.85(1.44)$ & $5.90(1.26)$ & $5.20(1.36)$ & $6.03(1.26)$ & $5.28(1.13)$ \\
\hline $\begin{array}{l}\text { Simvastatin daily dose } \\
\text { (mg) (mean SD) }\end{array}$ & 29(19) & $29(20)$ & $27(16)$ & $35(27)$ & $28(18)$ & $33(25)$ & $27(18)$ & $26(17)$ & $27(16)$ & $30(24)$ & $28(19)$ & $30(22)$ \\
\hline \multicolumn{13}{|l|}{ Social economic status } \\
\hline 1 (most deprived) & 285(46.1) & $126(43.2)$ & $141(20.3)$ & $50(28.6)$ & $46(25.4)$ & $18(30.0)$ & $108(25.2)$ & $106(28.5)$ & $1015(25.5)$ & $125(24.9)$ & $837(18.7)$ & $322(25.1)$ \\
\hline $2-4$ & $254(42.1)$ & $130(45.2$ & $402(58.9)$ & $90(52.6)$ & 100(56.8) & $35(58.3)$ & 244(56.9) & 210(56.9) & $2275(57.1)$ & $310(61.6)$ & $2681(59.8)$ & $760(59.1)$ \\
\hline 5 (most affluent) & $64(10.4)$ & $31(10.6)$ & 139(20.0) & $31(17.7)$ & $30(16.6)$ & $7(11.7)$ & $77(18.0)$ & $56(15.1)$ & $693(17.4)$ & $68(13.5)$ & $964(21.5)$ & 203(15.8) \\
\hline \multicolumn{13}{|l|}{ Concurrent use of drugs } \\
\hline Analgesics & $453(73.4)$ & $222(76.0)$ & $591(84.9)$ & $156(89.1)$ & $153(84.5)$ & $55(91.7)$ & $303(68.6)$ & $279(72.3)$ & 2293(55.9) & $335(65.2)$ & 1897(41.5) & $666(50.7)$ \\
\hline Positive inotropic drugs & $30(4.9)$ & $49(16.8)$ & $17(2.4)$ & $6(3.4)$ & $3(1.7)$ & $7(11.7)$ & $40(9.1)$ & $89(23.1)$ & $174(4.2)$ & $76(14.8)$ & $106(2.3)$ & $62(4.7)$ \\
\hline Diuretics & $386(62.6)$ & 183(62.7) & $416(59.8)$ & $100(57.1)$ & $97(53.6)$ & $39(65.0)$ & $313(70.8)$ & $311(80.6)$ & 1932(47.1) & $325(63.2)$ & $2082(45.5)$ & $569(43.3)$ \\
\hline $\begin{array}{l}\text { Beta-adrenoceptor } \\
\text { blocking drugs }\end{array}$ & $97(15.7)$ & $78(26.7)$ & $290(41.7)$ & $94(53.7)$ & $57(31.5)$ & $41(68.3)$ & 204(46.2) & 208(53.9) & 1456(35.5) & 299(58.2) & 1816(39.7) & 776(59.0) \\
\hline $\begin{array}{l}\text { Hypertension and } \\
\text { heart failure }\end{array}$ & $373(60.5)$ & 213(73.0) & $431(61.9)$ & 124(70.9) & $82(45.3)$ & $48(80.0)$ & 303(68.6) & $272(70.5)$ & $2712(66.1)$ & 390(75.9) & 2135(46.7) & $781(59.4)$ \\
\hline $\begin{array}{l}\text { Nitrates \& calcium-channel } \\
\text { blockers }\end{array}$ & $393(63.7)$ & 235(80.5) & 415(59.6) & $130(74.3)$ & $99(54.7)$ & $51(85.0)$ & $301(68.1)$ & $302(78.2)$ & 1951(47.6) & 390(72.9) & $2202(48.1)$ & $947(72.0)$ \\
\hline Anticoagulants & $43(7.0)$ & 49(16.8) & $56(8.1)$ & $13(7.4)$ & $13(7.2)$ & $13(21.7)$ & $64(14.5)$ & 104(26.9) & $237(5.8)$ & 103(20.0) & $227(4.9)$ & $97(7.4)$ \\
\hline Antiplatelet drugs & $400(64.8)$ & 244(83.6) & $423(60.8)$ & $152(86.9)$ & $93(51.4)$ & $45(75.0)$ & $242(54.8)$ & 297(76.9) & 2039(49.7) & $421(81.9)$ & 2329(50.9) & $1107(84.2)$ \\
\hline Corticosteroids & $457(74.1)$ & 210(71.9) & 208(29.9) & $46(26.3)$ & $109(60.2)$ & $32(53.3)$ & 163(36.9) & $127(32.9)$ & 1093(26.7) & $148(28.8)$ & $942(20.6)$ & 260(19.8) \\
\hline NSAID drugs & $180(29.2)$ & 63(21.6) & $342(49.1)$ & $71(40.6)$ & $110(60.8)$ & $34(56.7)$ & $91(20.6)$ & $77(20.0)$ & 1586(38.7) & $166(32.3)$ & 1292(28.3) & $323(24.6)$ \\
\hline \multicolumn{13}{|l|}{ Co-morbidity } \\
\hline Angina, TIA, heart failure & $56(9.1)$ & 142(48.6) & $34(4.9)$ & $17(9.7)$ & $6(3.3)$ & $9(15.0)$ & $33(7.5)$ & 140(36.3) & $169(4.1)$ & $109(21.2)$ & $187(4.1)$ & 508(38.6) \\
\hline Diabetes mellitus & $111(18.0)$ & 33(11.3) & 131(18.8) & $14(8.0)$ & $36(19.9)$ & $11(18.3)$ & $67(15.2)$ & $60(15.5)$ & - & - & - & - \\
\hline
\end{tabular}



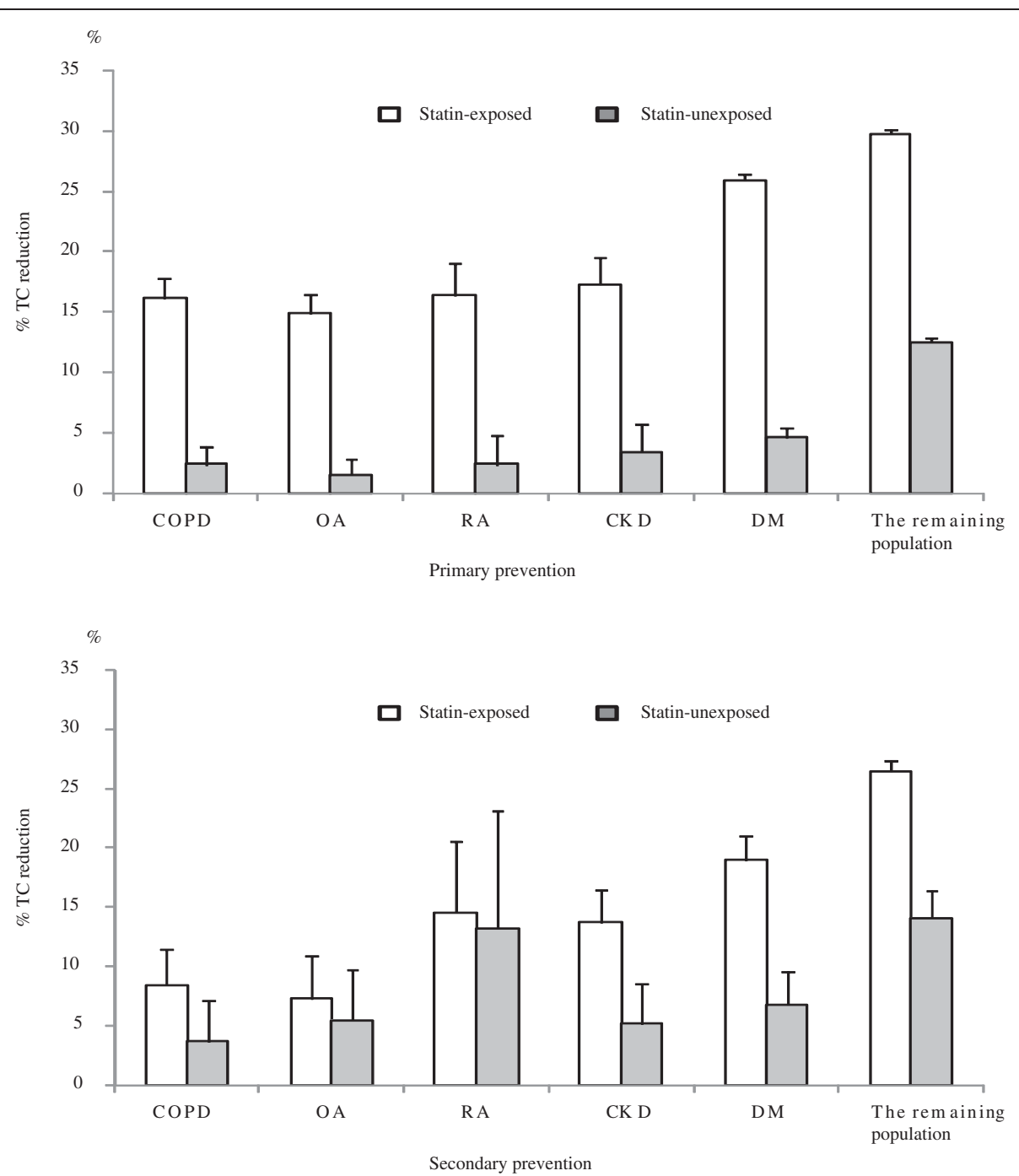

Figure 1 TC concentration changes in primary prevention and secondary prevention patients.

chronic disease patients for the primary prevention of $\mathrm{CV}$ events and in COPD, CKD, or DM patients for the secondary prevention of $\mathrm{CV}$ events. The risk reductions in APTC events and all-cause mortality in PP were significantly different across chronic disease patients and the rest of the population, but were not heterogeneous in SP.

The majority of previous studies focused on LDL-C concentration when evaluating the effect of statins in disease population. In this study, total cholesterol rather than LDL-C concentration was used to investigate the effect of statins on cholesterol changes in different chronic disease populations. There were several reasons: firstly, in the UK clinicians usually make statin titration decisions based on TC plus or minus HDL-C measurements. Secondly, LDL-C can be measured from the blood but it is expensive. So LDL-C is rarely measured in clinical practice and is usually calculated instead. A mathematical equation called Friedewald equation
$(\mathrm{LDL}=\mathrm{TC}-\mathrm{HDL}-\mathrm{TG} / 2.17(\mathrm{mmol} / \mathrm{L})$ is used to calculate LDL-C using values for total cholesterol, HDL-C and TG. When calculating LDL-C with the equation, it requires a fasting TG measurement [30]. Thirdly, in Tayside population there were approximately $15 \%$ of patients (COPD, OA, RA, or CKD) with at least two separate TC measurements and 35\% in diabetic patients. HDL-C concentration had similar measurements as TC concentration in MEMO database. However, there were very few TG measurements in these populations. This resulted in a large number of missing records for calculating LDL-C concentration and the effect of statins on LDL-C could not be investigated in this study.

In chronic disease patients exposed to statins, baseline $\mathrm{TC}$ concentrations were lower than those in the rest of the population. There are several possible explanations for this. Firstly, the average age in the chronic disease groups was higher than those in the rest of the 


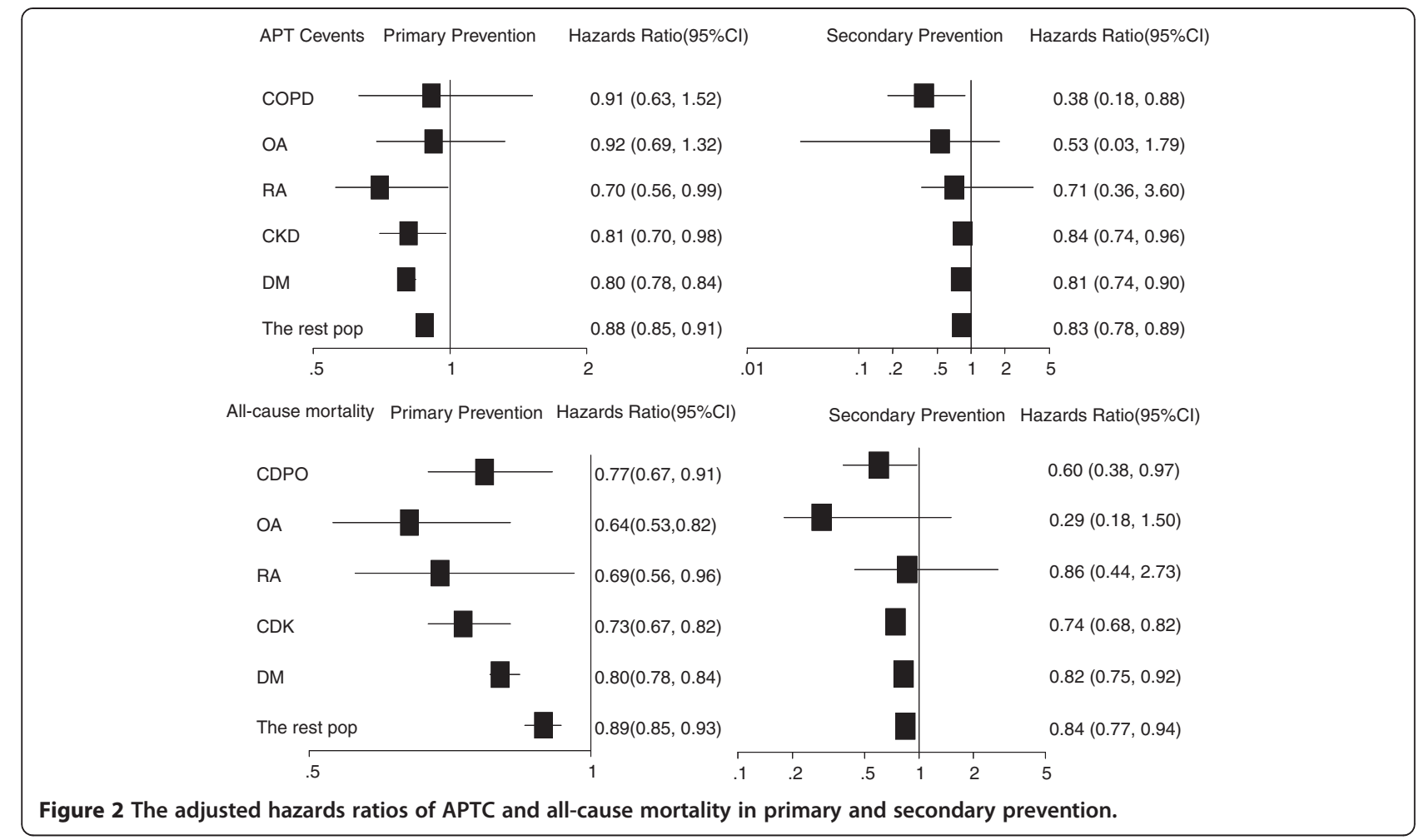

population in SP $(\mathrm{p}<0.001)$, and cholesterol concentrations decrease with age in older men and women $[31,32]$. Secondly, the rest of the population group was defined as population with at least two different TC measurements. These people were more likely to have other medical conditions such as hyperlipidmia, MI, and possibly to have higher baseline TC than the general population. So they were not completely representative of the general population. Thirdly, decreased TC concentration is seen with chronic disease or inflammation in the elderly [33-40]. Fourthly, low TC concentration is found with malnutrition or poor health status in elderly persons $[33,35]$. This appears to be related to lower baseline TC in SP than in PP. Fifthly, it could be also due to greater tendency of physicians to begin statins therapy earlier in patients with several risk factors of $\mathrm{CV}$ events. Finally, different mechanisms in chronic diseases may lead to different baseline TC and different TC reductions after statin therapy.

In addition, the impact of statins in chronic disease patients was less than that in the general population. One explanation was likely that the baseline TC concentrations did not reflect usual levels in these disease populations. The timing of lipid estimations can affect the results sometimes quite markedly. Cholesterol tests include fasting and non-fasting (random) blood samples. A recent study compared fasting and non-fasting TC and HDL-C concentration in adults and found that there were statistically significant differences between fasting and nonfasting results for total cholesterol, but no significant difference between non-fasting HDL-C and fasting HDL-C [41]. TC concentrations were slightly higher in the non-fasting state, but fasting and non-fasting values were highly correlated [41]. Therefore it is likely that many of lipid collections were non-fasting and performed during inter current illness, which result in different responses to statins in these chronic disease patients. Although there was a difference in TC, it was not clinically significant in diabetic or non-diabetic patients [42].

Average daily doses of statins in chronic disease groups were similar to those in the rest of the population in both PP and in SP. This suggested that differential $\mathrm{TC}$ reductions with statin use across the disease groups were not due to different statin doses. In secondary prevention there was no heterogeneity in the risk reduction across different study populations. This might be because the data were sparse in some chronic diseases with resulting low statistical power. In addition, the heterogeneous effects of standardized dose of simvastatin may be affected by the type of lipid abnormality and heterogeneity in differing disease states. For example, diabetic dyslipidemia consists of low HDL-C concentrations, increased TG concentrations, and postprandial lipemia, which is not captured by a focus on TC concentration [43]. In CKD patients, depressed HDL-C and increased TG are the major lipid abnormalities and LDL-C showed a 'J-curve' with respect to severity of disease 


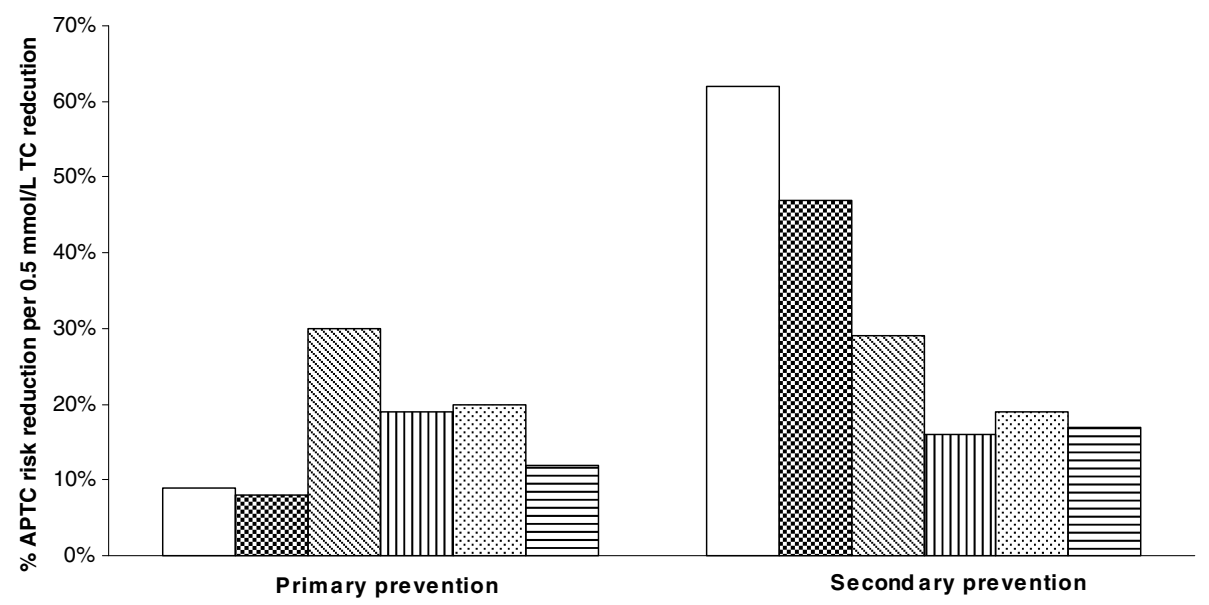

$\square C O P D \otimes O A \otimes R A \square C K D \otimes D M E$ The remaining population

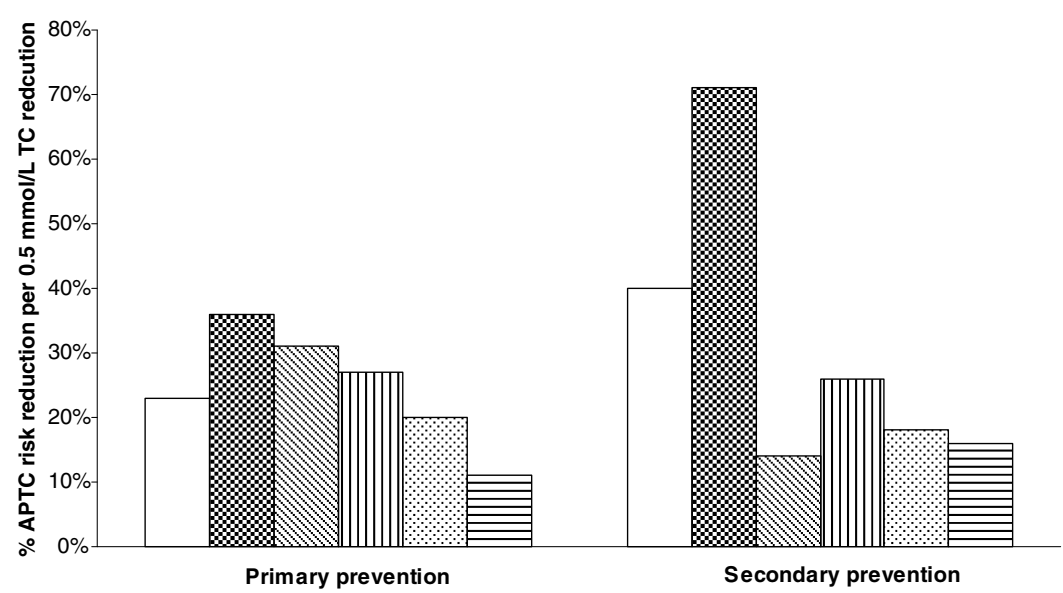

Figure 3 The proportional risk reduction of APTC events and all-cause mortality per $0.5 \mathrm{mmol} / \mathrm{L}$ TC concentration reduction.

[44]. Some evidence suggests that LDL-C is not increased and LDL-C is not strongly correlated with outcome in CKD [45].

Our study found that although there was less TC reduction with statins in some chronic disease groups than those in the rest of population, more benefit on the risk reduction of the outcomes was observed in some chronic disease groups, perhaps reflecting differential impacts of the pleiotropic anti-inflammatory effect of statins in chronic diseases. For example, statins have been suggested to have pleiotropic (anti-inflammatory) effects in patients with inflammatory diseases such as COPD and rheumatoid arthritis [46-48].

The beneficial effects of statins have been seen in clinical trials and observational studies in COPD, RA, CKD and diabetes [49-57]. Mancini et al reported that statin use exhibited a reduced MI risk ratio (RR 0.48 95\% CI 0.39-0.59) in COPD patients with coronary revascularization (high CV risk cohort) [49]. Daily use of $20 \mathrm{mg}$ or $40 \mathrm{mg}$ simvastatin was associated with the range from
$18 \%$ to $24 \%$ TC reduction and exhibited an improvement in vascular function in RA patients [51-53]. A metaanalysis that examined fifty RCTs and found that TC concentration was significantly lower by $19 \%$ with statins than with placebo in CKD patients with established CVD [56]. A 21\% risk reductions with lipid-lowering drugs in both incident and recurrent major coronary events in diabetic patients has been reported in another meta-analysis [57]. TC concentration showed a decrease of $15-20 \%$ in diabetic groups, which was a little smaller than that in our findings. In general, although there were some differences in the complexity of study design and patients selection between these studies and ours, they were in general agreements with our findings in patients with COPD or CKD or diabetes mellitus.

This is the first population-based study to investigate the comparative effectiveness of statins across chronic diseases patients and the rest of the population.While some randomized clinical trials provided the evidence of statin efficacy in an ideal setting in these chronic disease 
patients, our study assessed the effectiveness of statins under usual care setting. The study by using healthcare utilization databases has good external validity. In addition, we took into account an extensive list of covariates in adjusting for potential confounders. However, there are some limitations in our study. Our study may be influenced by potential unmeasured or immeasurable confounders such as cofounders related to the disease inherent progress or some other confounders of smoking status and alcohol consumption. We included the effect of statins on cholesterol lowering in five chronic diseases, but we did not study other chronic diseases such as chronic hepatitis, autoimmune diseases, etc. Another limitation of our study is the relatively small number of OA or RA patients with prior CV disease and resulting limited statistical power to provide meaningful results. We assumed if a patient dispensed his/her statin prescription he/she would be adherent to the treatment. However, we have no way of knowing true adherence. Furthermore comparative effectiveness studies in larger populations are required to confirm these relationships (e.g. the Trial of Atorvastatin for the primary prevention of cardiovascular events in patients with rheumatoid arthritis (TRACE-RA)). Also the effects of statins were not separately studied for men and women in our study. Gender differences might help to explain differential effects of statins in chronic diseases. Adherence and persistence of statins might also affect the effectiveness of statins and could vary between chronic diseases. Further studies should also study individual statins, smoking status, alcohol consumption, and other possible confounders in different populations.

\section{Conclusion}

The cholesterol lowering effect of statins among chronic diseases patients was generally less than that in the rest of the study population and showed considerable heterogeneity. The proportional risk reduction in cardiovascular events and all-cause mortality with statins per $0.5 \mathrm{mmol} / \mathrm{L}$ TC reduction also varied across different chronic disease groups compared with the rest of the population in PP, but there was no heterogeneity in SP. There was also an apparent disconnection between reductions in TC and outcome benefits that warrants further study. For practising clinicians, there appears to be meaningful heterogeneity in the effectiveness of statins between different disease groups which warrants further study.

\section{Additional file}

Additional file 1: Appendix 1.The crude event rates for the outcomes in chronic diseases patients and the rest of the population. Appendix 2. The adjusted hazards ratios of outcomes with statin use in PP and SP.

\section{Competing interests}

All authors declare: no support from any organisation for the submitted work; no financial relationships with any organisations that might have an interest in the submitted work in the previous 3 years; no other relationships or activities that could appear to have influenced the submitted work.

\section{Authors' contribution}

XS carried out the statistical analysis under the supervision of LW and wrote the first draft of the paper. LW, MJM, TMM were involved in the design of the study, interpretation of results and re-drafting of the paper. LW is guarantor for this study. All authors read and approved the final manuscript.

\section{Acknowledgement}

None.

\section{Author details}

${ }^{1}$ Medicines Monitoring Unit, Division of Medical Sciences, Ninewells Hospital \& Medical School, University of Dundee, Dundee DD1 9SY, UK. ²Department of Biochemical Medicine, Division of Clinical \& Population Sciences \& Education, Ninewells Hospital \& Medical School, University of Dundee, Dundee DD1 9SY, UK.

Received: 9 December 2011 Accepted: 14 August 2012

Published: 30 August 2012

\section{References}

1. Yach D, Hawkes C, Gould CL, Hofman KJ: The global burden of chronic diseases: Overcoming impediments to prevention and control. JAMA 2004, 291:2616-2622.

2. Beaglehole R, Yach D: Globalisation and the prevention and control of non-communicable disease: The neglected chronic diseases of adults. Lancet 2003, 362:903-908.

3. Halbert RJ, Natoli JL, Gano A, Badamgarav E, Buist AS, Mannino DM: Global burden of COPD: systematic review and meta-analysis. Eur Respir J 2006, 28:523-532.

4. Mathers CD, Loncar D: Projections of global mortality and burden of disease from 2002 to 2030. PLoS Med 2006, 3:e442.

5. Murray CJL, Lopez AD: Alternative projections of mortality and disability by cause 1990-2020: Global Burden of Disease Study. Lancet 1997, 349:1498-1504.

6. Wild S, Roglic G, Green A, Sicree R, King H: Global prevalence of diabetes: estimates for 2000 and projections for 2030. Diabetes Care 2004, 27:1047-1053.

7. Couser WG, Shah S, Kopple J, Beerkens P, Wilson A, Feehally J, Reiser T, Riella M: A call to action on World Kidney Day, 8 March 2007. Kidney Int 2007, 71:369-370.

8. Zhang QL, Rothenbacher D: Prevalence of chronic kidney disease in population-based studies: systematic review. BMC Publ Health 2008, 8:117.

9. Silman AJ, Pearson JE: Epidemiology and genetics of rheumatoid arthritis. Arthritis Res 2002, 4(Suppl 3):S265-S272.

10. Osteoarthritis: National clinical guideline for care and management in adults: Royal College of Physicians; Available from: http://www.nice.org.uk/ nicemedia/pdf/CG059FullGuideline.pdf (accessed on 01/03/2011).

11. Brion PH, Kalunian KC: Oxford textbook of medicine. In Section 18.8. Osteoarthritis. 4th edition. Edited by Warrell DA, Cox TM, Firth JD, Benz EJ Jr. Oxford: Oxford University; 2003.

12. Grundy SM, Benjamin IJ, Burke GL, Chait A, Eckel RH, Howard BV, Mitch W, Smith SC Jr, Sowers JR: Diabetes and cardiovascular disease: a statement for healthcare professionals from the American Heart Association. Circulation 1999, 100:1134-1146.

13. Haffner SM, Lehto S, Rönnemaa T, Pyörälä K, Laakso M: Mortality from coronary heart disease in subjects with type 2 diabetes and in nondiabetic subjects with and without prior myocardial infarction. $N$ Engl J Med 1998, 339:229-234.

14. Sarnak MJ, Levey AS, Schoolwerth AC, Coresh J, Culleton B, Hamm LL, McCullough PA, Kasiske BL, Kelepouris E, Klag MJ, Parfrey P, Pfeffer M, Raij L, Spinosa DJ, Wilson PW: Kidney disease as a risk factor for development of cardiovascular disease: a statement from the American Heart Association Councils on Kidney in Cardiovascular Disease, High Blood Pressure Research, Clinical Cardiology, and Epidemiology and Prevention. Circulation 2003, 108:2154-2169. 
15. Perazella MA, Khan S: Increased mortality in chronic kidney disease: a call to action. Am J Med Sci 2006, 331:150-153.

16. Sidney S, Sorel M, Quesenberry CP Jr, DeLuise C, Lanes S, Eisner MD: COPD and incident cardiovascular disease hospitalizations and mortality: Kaiser Permanente Medical Care Program. Chest 2005, 128:2068-2075.

17. Mannino DM, Thorn D, Swensen A, Holguin F: Prevalence and outcomes of diabetes, hypertension and cardiovascular disease in COPD. Eur Respir J 2008, 32:962-969.

18. Wallberg-Jonsson S, Ohman ML, Dahlqvist SR: Cardiovascular morbidity and mortality in patients with seropositive rheumatoid arthritis in Northern Sweden. J Rheumatol 1997, 24:445-451.

19. Maradit-Kremers H, Nicola PJ, Crowson CS, Ballman KV, Gabriel SE: Cardiovascular death in rheumatoid arthritis: a population-based study. Arthritis Rheum 2005, 52:722-732.

20. Sin DD, Anthonisen NR, Soriano JB, Agusti AG: Mortality in COPD: Role of comorbidities. Eur Respir J 2006, 28:1245-1257.

21. Yerram P, Karuparthi PR, Hesemann L, Horst J, Whaley-Connell A: Chronic kidney disease and cardiovascular risk. J Am Soc Hypertens 2007, 1:178-184.

22. Haffner SM: Management of dyslipidemia in adults with diabetes. American Diabetes Association. Diabetes Care 1998, 21:179-182.

23. Mallamaci F, Zoccali C, Tripepi G, Fermo I, Benedetto FA, Cataliotti A Bellanuova I, Malatino LS, Soldarini A: Hyperhomocysteinemia predicts cardiovascular outcomes in hemodialysis patients. Kidney Int 2002, 61:609-614

24. Hunninghake DB: Cardiovascular disease in chronic obstructive pulmonary disease. Proc Am Thorac Soc 2005, 2:44-49.

25. Baigent C, Blackwell L, Emberson J, Holland LE, Reith C, Bhala N, Peto R, Barnes EH, Keech A, Simes J, Collins R: Cholesterol Treatment Trialists' (CTT) collaboration. Efficacy and safety of more intensive lowering of LDL cholesterol: a meta-analysis of data from 170,000 participants in 26 randomised trials. Lancet 2010, 376:1670-1681.

26. Murphy MJ, Wei L, Watson AD, MacDonald TM: 'Real-life' reduction in cholesterol with statins, 1993 to 2002. Br J Clin Pharmacol 2008, 65:587-592.

27. Sheng $X$, Wei L, Murphy MJ, MacDonald TM: Statins and total (not LDL) cholesterol concentration and outcome of myocardial infarction: results from a meta-analysis and an observational study. Eur J Clin Pharmacol 2009, 65:1071-1080.

28. The Scottish Government: Scottish Diabetes Core Dataset:; . Available from: http://www.scotland.gov.uk/Publications/2003/01/16290/17641. (accessed on 30/03/2011).

29. Higgins JP, Thompson SG: Quantifying heterogeneity in a meta-analysis. Stat Med 2002, 21(11):1539-1558.

30. Friedwald WT, Levy RI, Fredrickson DS: Estimation of the concentration of low-density lipoprotein cholesterol in plasma, without use of the preparative ultracentrifuge. Clin Chem 1972, 18:499-502.

31. Ferrara A, Barrett-Connor E, Shan J: Total, LDL, and HDL cholesterol decrease with age in older men and women. The Rancho Bernardo Study 1984-1994. Circulation 1997, 96:37-43.

32. Abbott RD, Yano K, Hakim AA, Burchfiel CM, Sharp DS, Rodriguez BL, Curb JD: Changes in total and high-density lipoprotein cholesterol over 10- and 20 year periods (the Honolulu Heart Program). Am J Cardio/ 1998, 82:172-178.

33. Manolio TA, Ettinger WH, Tracy RP, Kuller LH, Borhani NO, Lynch JC, Fried LP: Epidemiology of low cholesterol levels in older adults. The Cardiovascular Health Study. Circulation 1993, 87:728-737.

34. Schaefer EJ, Moussa PB, Wilson PW, McGee D, Dallal G, Castelli WP: Plasma lipoproteins in healthy octogenarians: Lack of reduced high density lipoprotein cholesterol levels: Results from the Framingham Heart Study. Metabolism 1989, 38:293-296.

35. Kalantar-Zadeh K, Ikizler TA, Block G, Avram MM, Kopple JD: Malnutritioninflammation complex syndrome in dialysis patients: causes and consequences. Am J Kidney Dis 2003, 42:864-881.

36. Iribarren C, Jacobs DR Jr, Sidney S, Claxton AJ, Gross MD, Sadler M Blackburn H: Serum total cholesterol and risk of hospitalization, and death from respiratory disease. Int J Epidemio/ 1997, 26:1191-1202.

37. Rantapää-Dahlqvist S, Wållberg-Jonsson S, Dahlén G: Lipoprotein (a), lipids, and lipoproteins in patients with rheumatoid arthritis. Ann Rheum Dis 1991, 50:366-368.

38. Dursunoğlu D, Evrengül H, Polat B, Tanriverdi H, Cobankara V, Kaftan A, Kiliç M: Lp(a) lipoprotein and lipids in patients with rheumatoid arthritis: serum levels and relationship to inflammation. Rheumatol Int 2005, 25:241-245.
39. Liu Y, Coresh J, Eustace JA, Longenecker JC, Jaar B, Fink NE, Tracy RP, Powe NR, Klag MJ: Association between cholesterol level and mortality in dialysis patients: Role of inflammation and malnutrition. JAMA 2004 291:451-459.

40. Anderson KM, Castelli WP, Levy D: Cholesterol and mortality. 30 years of follow-up from the Framingham study. JAMA 1987, 257:2176-2180.

41. Craig SR, Amin RV, Russell DW, Paradise NF: Blood cholesterol screening. J Gen Intern Med 2000, 15:395-399.

42. Weiss $R$, Harder $M$, Rowe J: The relationship between nonfasting and fasting lipid measurments in patients with or without type 2 diabetes mellitus receiving treatment with 3-hydroxy-3methyglutaryl-coenzyme A reductase inhibitors. Clin Ther 2003, 25:1490-1497.

43. American Diabetes Association: Management of dyslipidemia in adults with diabetes. Diabetes Care 1998, 21(1):179-182.

44. Harper CR, Jacobson TA: Managing dyslipidemia in chronic kidney disease. J Am Coll Cardiol 2008, 51(25):2375-2384.

45. Kaysen GA: Dyslipidemia in chronic kidney disease: causes and consequences. Kidney Int 2006, 70:555-558.

46. Walsh GM: Statins as emerging treatments for asthma and chronic obstructive pulmonary disease. Expert Rev Respir Med 2008, 2(3):329-335.

47. McCarey DW, Mclnnes IB, Madhok R, Hampson R, Scherbakov O, Ford I, Capell HA, Sattar N: Trial of Atorvastatin in Rheumatoid Arthritis (TARA): doubleblind, randomised placebo-controlled trial. Lancet 2004, 363(9426):2015-2021.

48. Paraskevas Kl: Statin treatment for rheumatoid arthritis: a promising novel indication. Clin Rheumatol 2008, 27(3):281-287.

49. Lee TM, Lin MS, Change NC: Usefulness of C-reactive protein and interleukin- 6 as predictors of outcomes in patients with chronic obstructive pulmonary disease receiving pravastatin. Am J Cardiol 2008 101:530-535.

50. van Gestel YR, Hoeks SE, Sin DD, Simsek C, Welten GM, Schouten O, Stam H, Mertens FW, van Domburg RT, Poldermans D: Effect of statin therapy on mortality in patients with peripheral arterial disease and comparison of those with versus without associated chronic obstructive pulmonary disease. Am J Cardiol 2008, 102(2):192-196.

51. Mäki-Petäjä KM, Booth $A D$, Hall FC, Wallace SM, Brown J, McEniery CM Wilkinson IB: Ezetimibe and simvastatin reduce inflammation, disease activity, and aortic stiffness and improve endothelial function in rheumatoid arthritis. J Am Coll Cardiol 2007, 50(9):852-858.

52. Hermann F, Forster A, Chenevard R, et al: Simvastatin improves endothelial function in patients with Rheumatoid arthritis. J Am Coll Cardiol 2005, 45:461-464.

53. Tikiz C, Utuk O, Pirildar T, Bayturan O, Bayindir P, Taneli F, Tikiz H, Tuzun C: Effects of Angiotensin-converting enzyme inhibition and statin treatment on inflammatory markers and endothelial functions in patients with longterm rheumatoid arthritis. J Rheumatol 2005, 32(11):2095-2101.

54. Van Doornum S, McColl G, Wicks IP: Atorvastatin reduces arterial stiffness in patients with rheumatoid arthritis. Ann Rheum Dis 2004, 63(12):1571-1575

55. Charles-Schoeman C, Khanna D, Furst DE, McMahon M, Reddy ST, Fogelman AM, Paulus HE, Park GS, Gong T, Ansell BJ: Effects of high-dose atorvastatin on antiinflammatory properties of high density lipoprotein in patients with rheumatoid arthritis: a pilot study. J Rheumatol 2007, 34(7):1459-1464.

56. Strippoli GF, Navaneethan SD, Johnson DW, Perkovic V, Pellegrini F, Nicolucci A, Craig JC: Effects of statins in patients with chronic kidney disease: meta-analysis and meta-regression of randomised controlled trials. BMJ 2008, 336(7645):645-651.

57. Costa J, Borges M, David C, Vaz Carneiro A: Efficacy of lipid lowering drug treatment for diabetic and non-diabetic patients: meta-analysis of randomised controlled trials. BMJ 2006, 332(7550):1115-1124.

doi:10.1186/1471-2458-12-712

Cite this article as: Sheng et al:: The comparative effectiveness of statin therapy in selected chronic diseases compared with the remaining population. BMC Public Health 2012 12:712. 